\title{
A Mixed Integer Programming Model for Coordination of Multi-Product Two-Echelon Supply Chain Using Meta-heuristic Solutions
}

Yahia Zare Mehrjerdi* and SH Anvar

Department of Industrial Engineering, Yazd University, Iran

\begin{abstract}
This paper investigates a two-level supply chain model in which a single supplier provides multiple products to a group of retailers. Integration and coordination between members of this two-echelon supply chain is considered and a mixed integer model is developed. The problem determines the timing and the amount of replenishment for each retailer as well as the type and the quantity of the products that must be carried by each vehicle. The objective of this article is to minimize the sum cost of vehicle, material handling and the inventory holding of the whole supply chain. At first, an exact approach is developed to solve the problem but due to the complexity of the model (known as NP-hard problem) this model could only be applied to small-sized problems. Then, two well-known meta-heuristic algorithms, GA and SA, are employed as solution techniques and some numerical examples are provided for illustration purposes. For small-sized problems, the results indicate that the developed GA and SA methods present near optimum solutions.
\end{abstract}

Keywords: SCM; Multi retailer; Multi product; Coordination; Genetic algorithm; Simulated annealing

\section{Introduction}

Nowadays, there are efforts to reduce the cost of the whole supply chain as well as determining the delivery time and the replenishment quantity. Some investigators focus on routing or determining the location of the facilities meanwhile minimizing the total cost of supply chain. In this paper, we focus on a two-level supply chain that the elements of that are comprised of a single supplier and multiple retailers. The goal of this investigation is to minimize the total cost of such supply chains simultaneously to determine the timing and the amount of replenishment as well as the type and the amount of each item

With regard to the system proposed here, we further assume that the members of supply chain are located in the regional territories and the facilities are relatively close to each other so that the distance won't affect the transportation cost significantly, and hence we just have a fixed vehicle cost to consider. When a vehicle leaves the supplier for the retailer's warehouse, it carries variety of products demanded by the retailers but limited by its capacity only.

Recent researches on supply chain management mostly focus on the coordination and integration issues among members of a supply chain such as manufacturers, distributors, wholesalers, and retailers. Arshinder et al. [1] reviewed research on the importance of supply chain coordination and presented various coordination issues and mechanisms. Information sharing among members of a supply chain is considered to be a useful tool for supply chain management. Sarmah et al. [2] investigated a coordination problem in a single-manufacturer and multiple heterogeneous buyers' situation. Two typical cases were explored there: (i) ex-site delivery case considering manufacturer dominance where manufacturer with larger production lot size delivers the item to the group of heterogeneous buyers at common replenishment time through common carrier and (ii) ex-factory delivery case with buyer's dominance and common replenishment time for delivery.

Jaber and Goyal [3] investigated the coordination of order quantities amongst the players in a three-level supply chain with a centralized decision process. The first level of the supply chain is consisted of multiple buyers, the second level of a vendor (e.g., manufacturer) while the third level is comprised of multiple suppliers. The model developed in that paper guarantees that the local costs for the players either remain the same as before coordination, or decrease as a result of such coordination.

Lee et al. [4] and $\mathrm{Wu}$ and Cheng [5] evaluated the benefits of information sharing in a supply chain, i.e., inventory reduction and cost savings. Sahin and Robinson [6] showed that the overall cost in a supply chain could be reduced by the information sharing with which the decisions for inventory replenishment and delivery planning are coordinated. In addition to that, Ryu et al. [7] presented methods for information sharing in supply chains and analyzed the effect on the performance of the supply chains in terms of throughput, inventory and service levels.

Bertazzi et al. [8] and Lee et al. [9] proposed decomposition approaches to integrate inventory and transportation planning problems with deterministic demands. On the other hand, for cases of stochastic demands, C-etinkaya and Lee [10] and Axsater [11] presented analytical results for the coordination problem between inventory and transportation decisions in VMI systems. In that model, a supplier has the autonomy to hold orders issued by retailers for some time instead of fulfilling them immediately. Meanwhile, Cetinkaya et al. [12] provided a stochastic model and an approximation method for an integrated inventory replenishment and delivery problem in a supply chain that consists of a supplier and a retailer with compound Poisson demands. Also, Xu and Leung [13] developed an inventory policy in a supply chain consisting of a vendor and a retailer in which there is a limit on the shelf space.

There are a number of research results on the inventory routing problem. For example, Viswanathan and Mathur [14], Kim and Kim [15,16] Bertazzi et al. [17], Kim et al. [18], and Campbell and Savelsbergh

*Corresponding author: Yahia Zare Mehrjerdi, Department of Industrial Engineering, Yazd University, Iran, E-mail: yazm2000@yahoo.com

Received February 12, 2013; Accepted March 26, 2013; Published April 01, 2013

Citation: Mehrjerdi YZ, Anvar SH (2013) A Mixed Integer Programming Model for Coordination of Multi-Product Two-Echelon Supply Chain Using Meta-heuristic Solutions. Ind Eng Manage 1: 107. doi:10.4172/2169-0316.1000107

Copyright: (c) 2013 Mehrjerdi YZ, et al. This is an open-access article distributed under the terms of the Creative Commons Attribution License, which permits unrestricted use, distribution, and reproduction in any medium, provided the original author and source are credited. 
[19] considered inventory routing problems with deterministic demands. On the other hand, Berman and Larson [20] and Kleywegt et al. [21] proposed solution methods for stochastic inventory routing problems.

Nachiappan et al. [22] in their paper handled the operational issues of a two-echelon single vendor-multiple buyers supply chain (TSVMBSC) model under vendor managed inventory (VMI) mode of operation. In order to find out the optimal sales quantity for each buyer in TSVMBSC problem, they formulated a mathematical model. Optimal sales price and acceptable contract price at different revenue share were subsequently derived with the optimal sales quantity. A genetic algorithm (GA) based heuristic was proposed to solve this TSVMBSC problem, which belonged to nonlinear integer programming problem (NIP). Lee and $\mathrm{Wu}$ [23] have discussed about the main causes of bullwhip effect and order batching. A simplified two-echelon supply chain system, with one supplier and one retailer that could choose different replenishment policies, was used as a demonstration. Two types of inventory replenishment methods were considered: the traditional methods (the event-triggered and the time-triggered ordering policies), and the statistical process control (SPC) based replenishment method. The results showed that the latter outperforms the traditional method in the categories of inventory variation, and in the number of backlog when the fill-rate of the prior model was set to be $99 \%$. That research provided a different approach to inventory cost-down other than the common methods like: information sharing, order batch cutting, and lead time reduction. By choosing a suitable replenishment policy, the number of backorder and the cost of inventory could be reduced.

Wang et al. [24] proposed an original method for evaluating complex supply chains. A tentative multi-echelon production, transportation and distribution system with stochastic factors built-in was employed as a test bed for the proposed method.

Khouja [25] formulated a three-stage supply chain model where a firm can supply many customers. They dealt with three inventory coordination mechanisms between chain members and solved a cost minimization model for each. Lee et al. [9] studied about the distribution planning model for the multi-level supply chain network. The objective function of suggested model was to minimize logistic costs such as replenishment cost, inventory holding cost and transportation cost. A mixed integer programming formulation and heuristics for practical use were suggested. Lee [9] modeled the distribution planning for the multi-level supply chain network. Products which are manufactured at factory were delivered to customers through warehouses and distribution centers for given customer demands. The objective function of suggested model was to minimize logistic costs such as replenishment cost, inventory holding cost and transportation cost.

Monthatipkul and Yenradee [26] proposed a new inventory control system called the inventory distribution plan (IDP) control system for one-warehouse and multi-retailer supply chain. In the IDP control system, a proposed mixed-integer linear programming model was solved to determine an optimal IDP that controls the inventories of the supply chain.

This paper is organized as follows: Decision model is given in Section 2. Section 3 is devoted to the branch and bound method as well as GA and SA that are developed as solution techniques. In section 4, proposed algorithms are compared using sample numerical example problems. The conclusion and future works explained in the last section.

\section{Decision Model}

A single supplier, multi retailer with multi-item decision model is the problem of concern that determines the replenishment quantity and timing. This means that the number of periods and amount of products that must be replenished as well as the type of products and the quantity of them that must be carried by the vehicles and delivered to retailers is determined by this model. The objective is minimizing the sum of fixed vehicle cost, retailer-dependent material handling cost, transportation cost and inventory holding cost of whole supply chain.

The model proposed here is based upon the following assumptions:

- Customer demands are known and fixed during each period

- Supplier is the leader and retailers are the followers. This means that supplier makes decision about the quantities and the timing of replenishment and determines the types and the amounts of products to be delivered to the retailers by the vehicles

- The number of each type of vehicle is enough to handle all delivery replenishments

- A vehicle can visit some retailers in a single trip

- Transportation cost consists of a fixed vehicle cost and a fixed retailer-dependent handling cost. A fixed vehicle cost is incurred when a vehicle used for delivery and a fixed retailerdependent material handling is incurred when a vehicle arrives in a retailer warehouse

- All kind of products have the same cubical space, and all the vehicles have equal capacity

For illustration purposes, a mixed integer programming model is formulated taking a single supplier, multiple retailers and multiple items into consideration. The notations and variables are as follows:

$i$ : Index for products $(i 1,2, \ldots .$, ;

: Index for retailers $(1,2, \ldots$, ;

: Index for periods $(1,2, \ldots$, ;

: Index for vehicles $(1,2, \ldots$, ;

ijt: Demand of product i by retailer in period;

ijt: Unit inventory holding cost for product $\mathrm{i}$, at retailer, at the end of period;

: Fixed vehicle cost;

: Fixed material handing cost for retailer when a vehicle visits that retailer;

: Loading capacity of a vehicle;

: A sufficiently large number;

${ }_{i j t}:$ Inventory level of product $\mathrm{i}$ in retailer at the end of period;

${ }_{i j k t}$ : Quantity delivered of product i to retailer by vehicle in period;

i.e., ${ }_{j k t j k t j k t \ldots}{ }_{j k t} \quad i j k t$

${ }_{k t}$ : Quantity delivered by vehicle in period;

${ }_{i k t}$ : Quantity of product $i$ delivered by vehicle in period;

i.e., ${ }_{j k t j k t j k t \ldots . .} k t{ }_{i}{ }^{i k t}{ }_{i}{ }_{j} i j k t$

${ }_{j k t}^{1} i_{j_{j h} \Sigma_{i j p t} 0}$ 


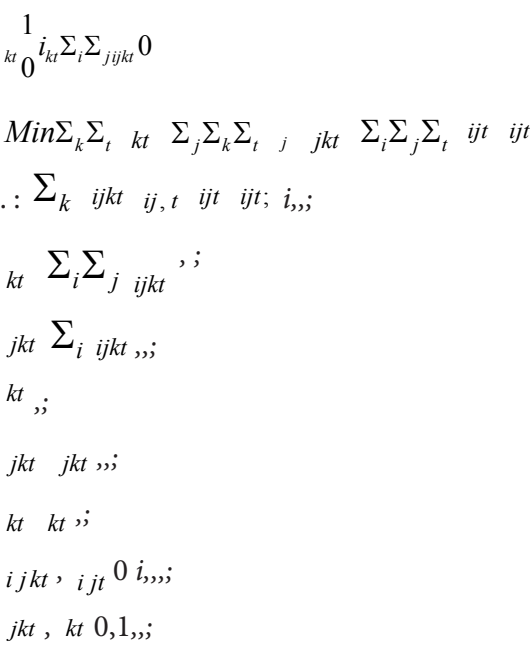

The objective function is comprised of three terms where the first one from the left denotes the sum of fixed vehicle cost and the second one illustrates the sum of retailer dependent material handling cost and the third shows the sum of inventory holding cost. Equation (4) shows that the inventory quantity in the current period for a product is equal to the sum of the newly arrived products plus the products remaining from the previous period minus the current demand. Equations (5) and (6) are the variables defined formerly. Constraint (7) ensures that the amount of delivered goods by a vehicle in a defined period should not exceed the loading capacity of that vehicle. Constraints (8) and (9) ensure that conditions (1) and (2) will be satisfied respectively. Constraints (10) and (11) are related to non-negative and binary conditions respectively.

\section{Solution Algorithm}

The mixed integer model developed in this research and shown in (3) to (11) is an NP-hard type problem. The developed proposed model is based upon the model studied in Kang et al [27]. Due to the fact that this is an NP-hard type problem we cannot find an exact solution for that and hence we develop two meta-heuristic algorithms, GA and SA, in this study. Thereafter, small-sized problems are solved using these meta-heuristic approaches and compare results with the branch and bound method.

\section{Genetic Algorithm}

With the conditions defined for the proposed model, a Genetic Algorithm is developed (figure 1). In our GA, the chromosomes generated randomly from initial population and represents the quantity of each products delivered by vehicles to each retailer in a defined period. The ${ }^{t}$ gene indicates the quantity of product $i$ that delivered to retailer by vehicle in period. A set of ten-digit binary numbers addresses one gene. Each chromosome is decoded to provide the feasible delivered quantity ${ }_{i j k}$ in integers by interpolating the gene information of the binary form with the following guidelines: Zero in all the nine digits would correspond to ${ }_{i j k t}$;

i.e.,

\begin{tabular}{|l|l|l|l|l|l|l|l|l|l|}
\hline 0 & 0 & 0 & 0 & 0 & 0 & 0 & 0 & 0 & 0 \\
\hline
\end{tabular}

And 'one' in all the nine digits would correspond to ${ }_{i j k t} 1023$;

i.e.,

\begin{tabular}{|l|l|l|l|l|l|l|l|l|l|}
\hline 1 & 1 & 1 & 1 & 1 & 1 & 1 & 1 & 1 & 1 \\
\hline
\end{tabular}

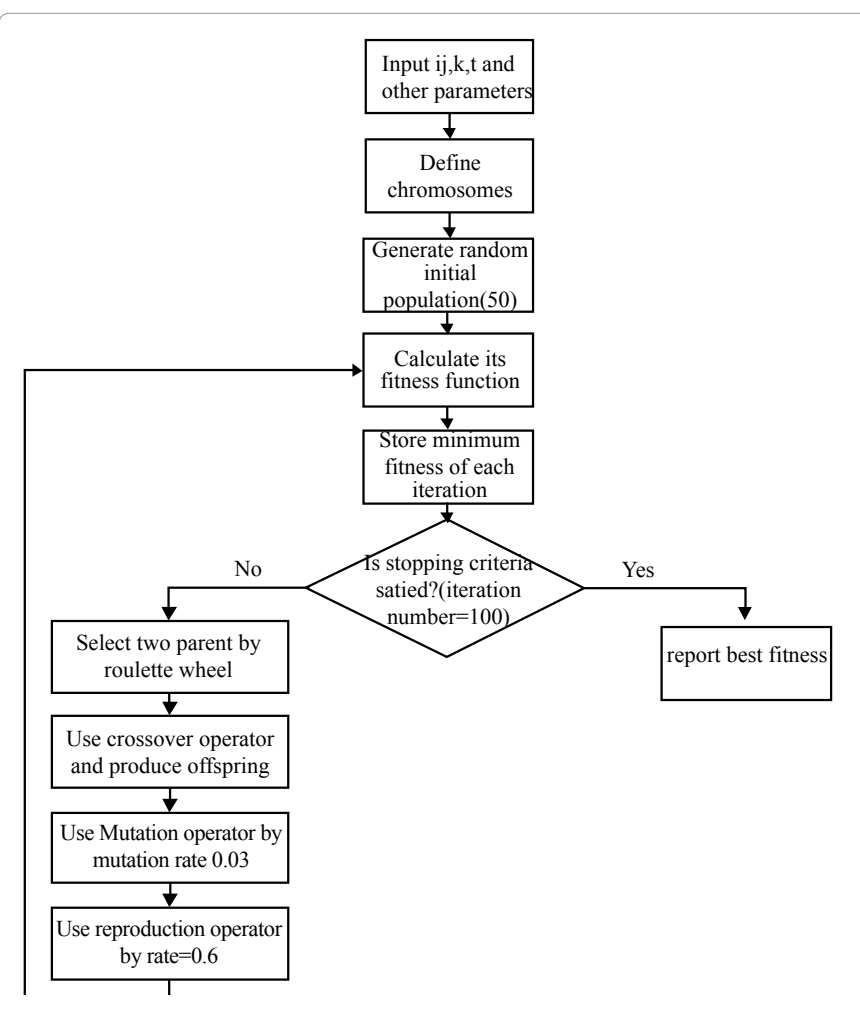

Figure 1: GA algorithm for decision model.

We know the size of population depends on the feasible solution space of the problem, which is normally dependent on the number of decision variables so in this study we assumed the population size equals 50 .

Minimization of channel cost is considered as fitness function. Each gene and every chromosome is tested or its fitness. The fitness parameter is the channel cost and is calculated using equation (3). Minimum fitness parameter is sorted out for each population and will be stored as the local best.

Value is sorted out after all iterations and it is stored as global best. The termination criterion is checked after sorting module to know whether the pre-determined number of iterations is completed. On its completion, the algorithm passes to the output module. Otherwise, the new set of population is generated as given in the new population generation module. The number of iterations depends upon the nature and the size of the problem and it is fixed by the user. The selection of the next population is based upon the survival probability, crossover to produce children and mutation to induce external influence in new generation. The next population of the same size is obtained with random selection (roulette wheel) procedure explained below. According to Roulett wheel we want to find out the best parents to survive new generation, so a random number is generated between 0 and 1 and a selected chromosome satisfies a condition in which the random number is less than cumulative probability of next chromosome and greater than cumulative probability of previous chromosome.

The selection process is repeated as many times as the population size. The method used here is reliable because it guarantees that the fittest individuals will be selected, and that the frequency of the fittest getting selected will be more. This procedure enables the fit chromosome to get the multiple copies and the worst to die. The value for population 
Citation: Mehrjerdi YZ, Anvar SH (2013) A Mixed Integer Programming Model for Coordination of Multi-Product Two-Echelon Supply Chain Using Meta-heuristic Solutions. Ind Eng Manage 1: 107. doi:10.4172/2169-0316.1000107

Page 4 of 6

rate is assumed as 0.6 , so at least $60 \%$ of chromosomes selected in the earlier selection module will undergo crossover operation and produce offspring. The purpose of mutation is for the introduction of new genetic material or for the recreation of good genes that were lost by chance through poor selection of mates. In this paper the mutation rate is assumed as 0.03 .

\section{Simulated Annealing}

We know the cooling schedule, in turn, is specified by:

- Initial and final temperature values;

- Updating function for changing the temperature

- $\quad$ Equilibrium test

The behavior of the simulated annealing algorithm depends upon the temperature. Perhaps the most important thing is who determines the initial temperature t0. In this paper, the initial temperature is set to 10. In theory, the simulated annealing procedure should be continued until final temperature $\mathrm{tf}$ gets close to zero but in practice other stopping criteria are also applied. Some of these criteria are:

- The value of the objective function has not decreased for a large

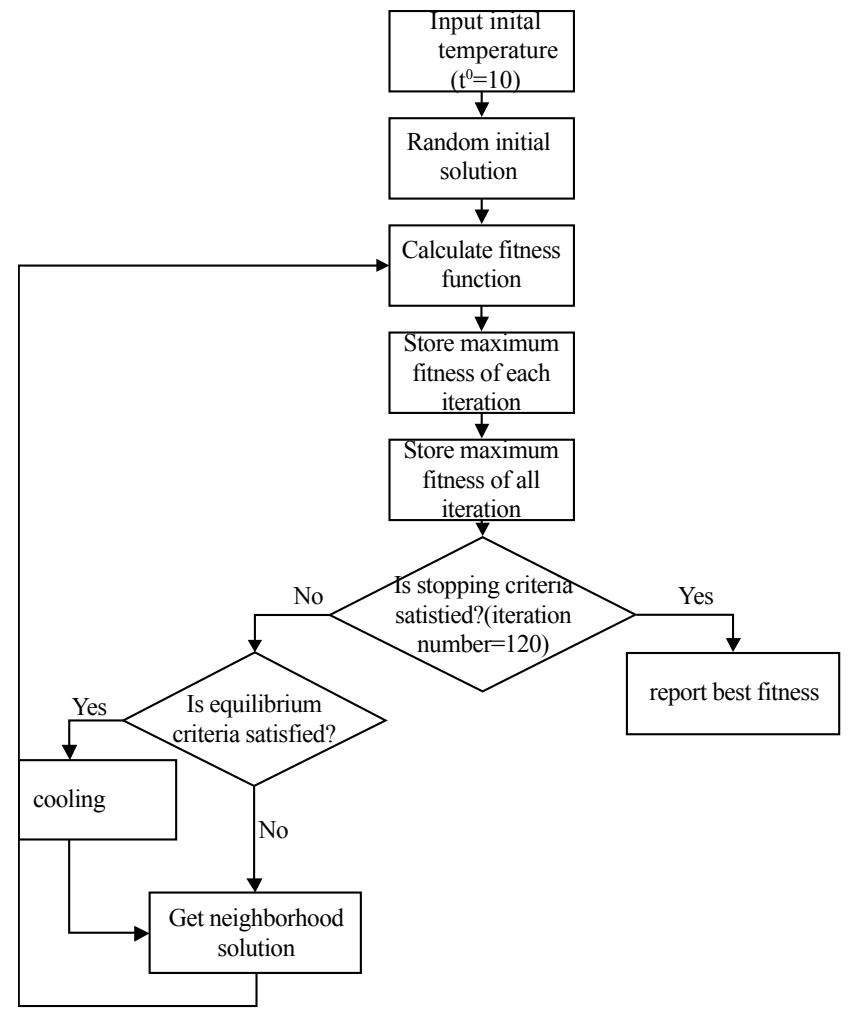

Figure 2: SA algorithm for decision model.

\begin{tabular}{|c|c|c|c|c|c|c|c|c|c|}
\hline $\mathrm{j}$ & $\mathrm{k}$ & $\mathrm{t}$ & Run 1 & Run 2 & Run 3 & Run 4 & Run 5 & Best result \\
\hline 2 & 2 & 2 & 2 & 8336.5000 & 8331.7000 & 8339.7000 & 8376.1000 & 8329.3000 & 8329.3000 \\
\hline 3 & 2 & 2 & 2 & 8304.8000 & 8242.4000 & 8219.3000 & 8228.1000 & 8352.5000 & 8219.3000 \\
\hline 5 & 2 & 2 & 2 & 9040.5200 & 9403.9000 & 8914.4190 & 9087.8230 & 10188.3110 & 8914.4190 \\
\hline 2 & 2 & 2 & 3 & 12269.0489 & 12269.0489 & 12269.0489 & 12269.0489 & 12269.0489 & 12269.0489 \\
\hline 2 & 3 & 2 & 3 & 15233.0451 & 15044.6670 & 15102.9840 & 15060.9660 & 15105.1990 & 15044.6670 \\
\hline
\end{tabular}

Table 1: Performance of GA algorithm for small-sized problem for 5 running times. number of consecutive trials

- The number of accepted moves has become less than a certain

- A fixed priori number of trials have been executed

In this paper, $100.9^{k}$ is used for decreasing temperature wherein is the iterations number.

For generating neighborhood solution, it is necessary to transform the basic solution into a binary form. Then, some binary values should randomly change from 0 to 1 and vice versa, and the number of bytes is changed depending on the problem type and size.

\section{Numerical Experiments}

To evaluate the performance of suggested algorithms we have designed some numerical example problems and compared the results of GA and SA algorithms (Figure 2) with the result of B\&B algorithm (exact solution for small-sized problems). For large-sized problems, we solved those problems only by GA and SA and compare them with each other. This is mainly because $\mathrm{B} \& \mathrm{~B}$ cannot solve large-sized problems in a reasonable amount of time.

The optimum solution can be used to find out the delivered quantities ${ }_{i j k t}$ for small-sized problems. Comparison between the results of GA, SA and B\&B (optimum solution) points to the closeness of results and hence suggesting the model's optimality of GA and SA. It should be considered that there are a lot of variables in our model even in small-sized problems; therefore we only consider the results.

We have generated some random problems the same as the following tables. Notations ( , and ( , illustrate the uniform distribution in range (, and discrete uniform distribution in range $($, , respectively. We have used the same assumptions as was used by Kang et al. [27] which are listed below:

- Demand quantity in a defined period was generated from $(100$ 500)

- Loading capacity of vehicles was set from this equation: $5 \Sigma_{i} \Sigma_{j}$ $\sum_{t i j t} l$

- The unit inventory cost was generated in $(1,10)$

- The retail-material handling cost was generated where $\Sigma_{i} \Sigma_{j} \Sigma_{t}$ ${ }_{i j t} /$ and $5 \Sigma_{i} \Sigma_{j} \Sigma_{t i j t} /$ and is generated from $(0.8,1.2)$.

- The fixed vehicle cost was set to. $\Sigma_{j j} /$ where is generated from $(0.8,1.2)$.

- The initial inventory was generated from $(0,200)$.

All algorithms were coded in MATLAB 7.0 and computational experiments were performed on a PC with

a Pentium 4 and $2.8 \mathrm{GHz}$ process speeds.

As shown in table 1, GA algorithm runs for 5 different problems small threshold for a large number of consecutive trials 
Citation: Mehrjerdi YZ, Anvar SH (2013) A Mixed Integer Programming Model for Coordination of Multi-Product Two-Echelon Supply Chain Using Meta-heuristic Solutions. Ind Eng Manage 1: 107. doi:10.4172/2169-0316.1000107

Page 5 of 6

\begin{tabular}{|l|l|l|l|l|l|l|l|c|c|}
\hline i & J & k & t & Run 1 & Run 2 & Run 3 & Run 4 & Run 5 & Best result \\
\hline 2 & 2 & 2 & 2 & 9202.1912 & 8863.5220 & 9780.0444 & 9577.0427 & 9239.7083 & 8863.5220 \\
\hline 3 & 2 & 2 & 2 & 8479.0310 & 8635.5382 & 8464.0153 & 8559.3621 & 8536.1759 & 8464.0153 \\
\hline 5 & 2 & 2 & 2 & 14972.5997 & 13746.7100 & 13137.6116 & 16204.1075 & 14368.2621 & 13137.6116 \\
\hline 2 & 2 & 2 & 3 & 13331.4730 & 12802.5034 & 13175.4595 & 13336.2703 & 13187.1352 & 12802.5034 \\
\hline 2 & 3 & 2 & 3 & 16156.0097 & 16461.6782 & 17197.7558 & 16169.2594 & 16451.0562 & 16156.0097 \\
\hline
\end{tabular}

Table 2: Performance of SA algorithm for small size problem for 5 running times.

\begin{tabular}{|c|c|c|c|c|c|c|c|c|}
\hline $\mathrm{i}$ & $\mathrm{j}$ & $\mathrm{k}$ & $\mathrm{T}$ & $\mathrm{GA}$ & GA Percentage Error (\%) & SA & SA Percentage Error (\%) & B \& B \\
\hline 2 & 2 & 2 & 2 & 8329.3000 & 0.0283 & 8863.5220 & 6.4439 & 8326.9460 \\
\hline 3 & 2 & 2 & 2 & 8219.3000 & 0.6034 & 8464.0153 & 3.5987 & 8170.0020 \\
\hline 5 & 2 & 2 & 2 & 8914.4190 & 0.3771 & 13137.6116 & 47.9306 & 8880.9290 \\
\hline 2 & 2 & 2 & 3 & 12269.0489 & 0.0000 & 12802.5034 & 4.34797 & 12269.0489 \\
\hline 2 & 3 & 2 & 3 & 15044.6670 & 8.0809 & 16156.0097 & 16.0648 & 13919.8200 \\
\hline
\end{tabular}

Table 3: Comparison between three algorithms.

\begin{tabular}{|c|c|c|c|c|c|}
\hline$j$ & $k$ & $t$ & GA & SA & NOS $=$ Min $(G A, S A)$ \\
\hline 5 & 5 & 5 & 23782.3413 & 25359.0092 & 23782.3413 \\
\hline 5 & 5 & 10 & 34392.2703 & 35673.1053 & 34392.2703 \\
\hline 5 & 10 & 5 & 27450.9024 & 29546.2745 & 27450.9024 \\
\hline 5 & 10 & 10 & 43608.3431 & 40783.5800 & 40783.5800 \\
\hline 10 & 5 & 5 & 17934.5748 & 18605.9690 & 17934.5748 \\
\hline 10 & 5 & 10 & 29626.9205 & 28648.7549 & 28648.7549 \\
\hline 10 & 10 & 5 & 42374.1300 & 45684.5563 & 42374.1300 \\
\hline
\end{tabular}

NOS = Near Optimum Solution

Table 4: Performance of GA and SA algorithms for fixed item number $(i=5)$.

\begin{tabular}{|c|c|c|c|c|c|}
\hline $\mathrm{i}$ & $\mathrm{k}$ & $\mathrm{t}$ & $\mathrm{GA}$ & $\mathrm{SA}$ & NOS*Min $(\mathrm{GA}, \mathrm{SA})$ \\
\hline 10 & 10 & 10 & 74688.7687 & 76117.4320 & 74688.7687 \\
\hline 10 & 10 & 20 & 91945.9097 & 91185.0080 & 91185.0080 \\
\hline 10 & 20 & 10 & 85369.7878 & 85447.1125 & 85369.7878 \\
\hline 10 & 20 & 20 & 102122.9768 & 104237.7650 & 102122.9768 \\
\hline 20 & 20 & 50 & 115047.3368 & 116800.3654 & 115047.3368 \\
\hline 50 & 50 & 50 & 161327.9875 & 170698.6549 & 161327.9875 \\
\hline
\end{tabular}

NOS = Near Optimum Solution

Table 5: Performance of GA and SA algorithms for fixed item number ( $j=15)$.

\begin{tabular}{|c|c|c|c|c|c|}
\hline $\mathrm{i}$ & $j$ & $\mathrm{t}$ & $\mathrm{GA}$ & $\mathrm{SA}$ & NOS*Min $(\mathrm{GA}, \mathrm{SA})$ \\
\hline 10 & 15 & 5 & 44365.5627 & 47258.2386 & 44365.5627 \\
\hline 10 & 20 & 10 & 80135.4859 & 79986.3275 & 79986.3275 \\
\hline 20 & 20 & 20 & 114875.2659 & 116898.1211 & 114875.2659 \\
\hline 20 & 30 & 30 & 137864.2568 & 141269.2416 & 137864.2568 \\
\hline 30 & 30 & 30 & 150102.1973 & 151233.4578 & 150102.1973 \\
\hline 30 & 40 & 20 & 143317.9866 & 148154.4762 & 143317.9866 \\
\hline 50 & 40 & 30 & 192322.4588 & 192579.9853 & 192322.4588 \\
\hline 50 & 50 & 50 & 227351.3324 & 231253.7954 & 227351.3324 \\
\hline
\end{tabular}

NOS $=$ Near Optimum Solution

Table 6: Performance of GA and SA algorithms for fixed vehicle number $(k=20)$.

and each problem is repeated 5 times, while table 2 is the results of SA runs for 5 different problems where each problem is repeated 5 times. In table 3, best solutions of GA and SA compared with the Branch and Bound method and we can show that results are close to the exact solution. Also, we have observed that GA outputs are better than the SA solutions. For large-sized problems, we have designed some problems with the results shown in tables 4 and 5. For these problems, although we don't have exact solutions, the minimum result of both meta-heuristic algorithms is selected as the best Near Optimum Solution (NOS).

As can be seen from tables 4-6 it is easier to solve problems with GA and SA algorithms to find near optimum solution. For large-sized problems, we must use these algorithms only due to the fact that exact solutions cannot be obtained in a reasonable amount of time. Also, it is clear that the results of GA algorithm are often better than the results of SA algorithm for such a class of problems. 
Citation: Mehrjerdi YZ, Anvar SH (2013) A Mixed Integer Programming Model for Coordination of Multi-Product Two-Echelon Supply Chain Using Meta-heuristic Solutions. Ind Eng Manage 1: 107. doi:10.4172/2169-0316.1000107

\section{Conclusion}

We considered a two level supply chain in which there is a single supplier and multiple retailers where supplier forecasts each retailer's demand and inventory level and tries to meet their needs. Supplier calculates the quantity and the timing of replenishment as well as the types of products and the quantity of each item. To accomplish this objective, supplier employs homogenous vehicles to distribute products among the retailers. Two meta-heuristic algorithms, GA and $\mathrm{SA}$, are developed for this mixed integer problem, and for small-sized numerical examples these two methods are compared with the branch and bound. It is easily seen that the results are proper. In addition to that, algorithms suggested here are used to solve some large scale problems and inferred that GA and SA gave relatively good solution in a reasonable amount of computational time. For future work, this research can be extended in several areas. As one of them, we suggest studying about multiple suppliers that provide services to a group of retailers.

\section{References}

1. Arshinder, Kanda A, Deshmukh SG (2008) Supply chain coordination: Perspectives, empirical studies and research directions. Int J Prod Econ 115: 316-335.

2. Sarmah SP, Acharya D, Goyal SK (2008) Coordination of a single-manufacturer/ multi-buyer supply chain with credit option. Int J Prod Econ 111: 676-685.

3. Jaber MY, Goyal SK (2008) Coordinating a three-level supply chain with multiple suppliers, a vendor and multiple buyers. Int J Prod Econ 116: 95-103.

4. Lee HL, So KC, Tang CS (2000) The value of information sharing in a two-level supply Chain. Manage Sci 46: 626-643.

5. Wu YN, Cheng TCE (2008) The impact of information sharing in a multipleechelon supply chain. Int J Prod Econ 115: 1-11.

6. Sahin F, Robinson Jr. EP (2005) Information sharing and coordination in maketo-order supply chains. J Oper Manag 23: 579-598.

7. Ryu S-J, Tsukishima T, Onari H (2009) A study on evaluation of demand information-sharing methods in supply chain. Int J Prod Econ 120: 162-175.

8. Bertazzi L, Paletta G, Spenranze MG (2005) Minimizing the total cost in an integrated vendor-managed inventory system. J Heuristics 11: 393-419.

9. Lee BK, Kang KH, Lee YH (2008) Decomposition heuristic to minimize total cost in a multi-level supply chain network. Computers and Industrial Engineering 54: 945-959.
10. Cetinkaya S, Lee C-Y (2000) Stock replenishment and shipment scheduling for vendor managed inventory systems. Manage Sci 46: 217-232.

11. Axsater S (2001) A note on stock replenishment and shipment scheduling for vendor-managed inventory systems. Manage Sci 47: 1306-1310.

12. Cetinkaya S, Tekin E, Lee C-Y (2008) A stochastic model for joint inventory and outbound shipment decisions. IIE Trans 40: 324-340.

13. Xu K, Leung MT (2009) Stocking policy in a two-party vendor managed channe with space restrictions. Int J Prod Econ 117: 271-285.

14. Viswanathan S, Mathur K (1997) Integrating routing and inventory decisions in one-warehouse multi-retailer multi-product distribution systems. Manage Sci 43: $294-312$

15. Kim JU, Kim YD (1999) A decomposition approach to a multi-period vehicle scheduling problem. Omega 27: 421-430.

16. Kim JU, Kim YD (2000) A Lagrangian relaxation approach to multi- period inventory/distribution planning. J Oper Res Soc 51: 364-370.

17. Bertazzi, L, Paletta G, Speranza MG (2002) Deterministic order-up-to leve policies in an inventory routing problem. Transport Sci 36: 119-132.

18. Kim JU, Kim YD, Shim SO (2002) Heuristic algorithms for a multi- period multistop transportation planning problem. J Oper Res Soc 53: 1027-1037.

19. Campbell AM, Savelsbergh MWP (2004) A decomposition approach for the inventory routing problem. Transport Sci 38: 488-502.

20. Berman O, Larson RC (2001) Deliveries in an inventory/routing problem using stochastic dynamic programming. Transport Sci 35: 192-213.

21. Kleywegt AJ, Nori VS, Savelsbergh MWP (2002) The stochastic inventory routing problem with direct deliveries. Transport Sci 36: 94-118.

22. Nachiappan SP, Jawahar N (2007) A genetic algorithm for optimal operating parameters of VMI system in a two-echelon supply chain. Eur J Oper Res 182 1433-1452.

23. Lee HT, Wu JC (2006) A study on inventory replenishment policies in a twoechelon supply chain system. Computers \& Industrial Engineering 51: 257-263.

24. Wang FF, Su CT (2007) Performance evaluation of a multi-echelon production transportation and distribution system: A matrix analytical approach. Eur J Ope Res 176: 1066-1083.

25. Khouja M (2003) Optimizing inventory decisions in a multi-stage multi-customer supply chain. Transport Res E-Log 39: 193-208.

26. Monthatipkul C, Yenradee P (2008) Inventory/distribution control system in a one-warehouse/ multi-retailer supply chain. Int J Prod Econ 114: 119-133.

27. Kang JH, Kim YD (2010) Coordination of inventory and transportation managements in a two-level supply chain. Int J Prod Econ 123: 137-145. 